\title{
Dynamic Multiscale Averaging (DMA) of Turbulent Flow
}

Richard W. Johnson

The INL is a

U.S. Department of Energy

National Laboratory

operated by

Battelle Energy Alliance

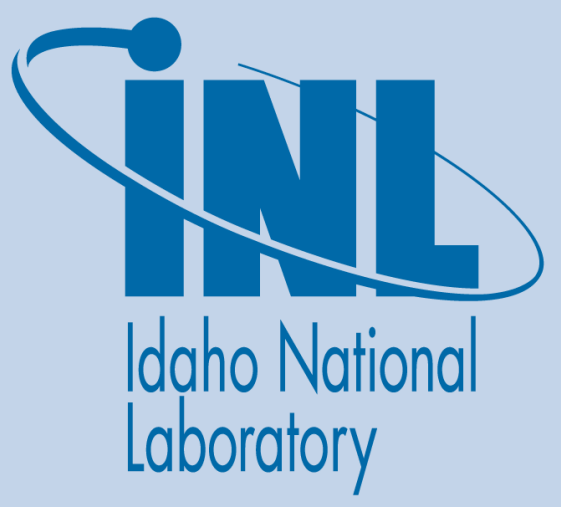

\section{September 2012}

The INL is a U.S. Department of Energy National Laboratory operated by Battelle Energy Alliance 


\section{DISCLAIMER}

This information was prepared as an account of work sponsored by an agency of the U.S. Government. Neither the U.S. Government nor any agency thereof, nor any of their employees, makes any warranty, expressed or implied, or assumes any legal liability or responsibility for the accuracy, completeness, or usefulness, of any information, apparatus, product, or process disclosed, or represents that its use would not infringe privately owned rights. References herein to any specific commercial product, process, or service by trade name, trade mark, manufacturer, or otherwise, does not necessarily constitute or imply its endorsement, recommendation, or favoring by the U.S. Government or any agency thereof. The views and opinions of authors expressed herein do not necessarily state or reflect those of the U.S. Government or any agency thereof. 


\section{Dynamic Multiscale Averaging (DMA) of Turbulent Flow}

Richard W. Johnson

September 2012

Idaho National Laboratory

Nuclear Energy Advanced Modeling \& Simulation Program

Idaho Falls, Idaho 83415

Prepared for the

U.S. Department of Energy

Office of Nuclear Energy

Under DOE Idaho Operations Office

Contract DE-AC07-05ID14517 



\section{SUMMARY AND RECOMMENDATIONS}

A new approach called dynamic multiscale averaging (DMA) for computing the effects of turbulent flow is described. The new method encompasses multiple applications of temporal and spatial averaging, that is, multiscale operations. Initially, a direct numerical simulation (DNS) is performed for a relatively short time; it is envisioned that this short time should be long enough to capture several fluctuating time periods of the smallest scales. The flow field variables are subject to running time averaging during the DNS. After the relatively short time, the timeaveraged variables are volume averaged onto a coarser grid. Both time and volume averaging of the describing equations generate correlations in the averaged equations. These correlations are computed from the flow field and added as source terms to the computation on the next coarser mesh. They represent coupling between the two adjacent scales. Since they are computed directly from first principles, there is no modeling involved. However, there is approximation involved in the coupling correlations as the flow field has been computed for only a relatively short time. After the time and spatial averaging operations are applied at a given stage, new computations are performed on the next coarser mesh using a larger time step. The process continues until the coarsest scale needed is reached. New correlations are created for each averaging procedure. The number of averaging operations needed is expected to be problem dependent.

The new DMA approach is applied to a relatively low Reynolds number flow in a square duct segment. The DNS computation is initiated using a nonphysical asymmetric initial condition. After five seconds of simulation time, running time averages are initiated and performed for another two seconds. The time-averaged variables are then volume averaged onto a coarser mesh. Figure S-1 illustrates the instantaneous stream-wise velocity at 7 seconds and the same velocity after time and volume averaging. The new coupling correlations are also computed. These correlations are assumed to be constant for succeeding computations and are added as source terms. Calculations are then performed using the time and volume-averaged field variables on the coarser grid. The coarser grid is essentially created using a mesh that has been reduced in each coordinate by a factor of four. The time step is increased by a factor of five. The compute time for a given time interval on the coarse mesh is about 1/500 the corresponding time required on the fine mesh. Figure S-2 illustrates the time-averaged stream-wise velocity and vorticity on the coarse mesh at 130 seconds. Time-averaged stream-wise velocity and vorticity contours appear to be very similar to a full DNS for a similar flow reported in the literature. Expected symmetry for the final results is produced for the DMA method. The results obtained indicate that DMA holds significant potential in being able to accurately compute turbulent flow without modeling for practical engineering applications.

The present study has been performed to demonstrate proof of principle. Significantly more research should be pursued to fully explore the effectiveness and efficiency of the method. 
Research should be performed to investigate how best to initiate turbulence to minimize the initial DNS calculation, how long the DNS should be performed, how much to scale-up for a given scaling, which numerical methods are best (fastest and most accurate) to use to perform DMA, how well the method performs for large Reynolds number flows and how the method can be applied to complex geometries.

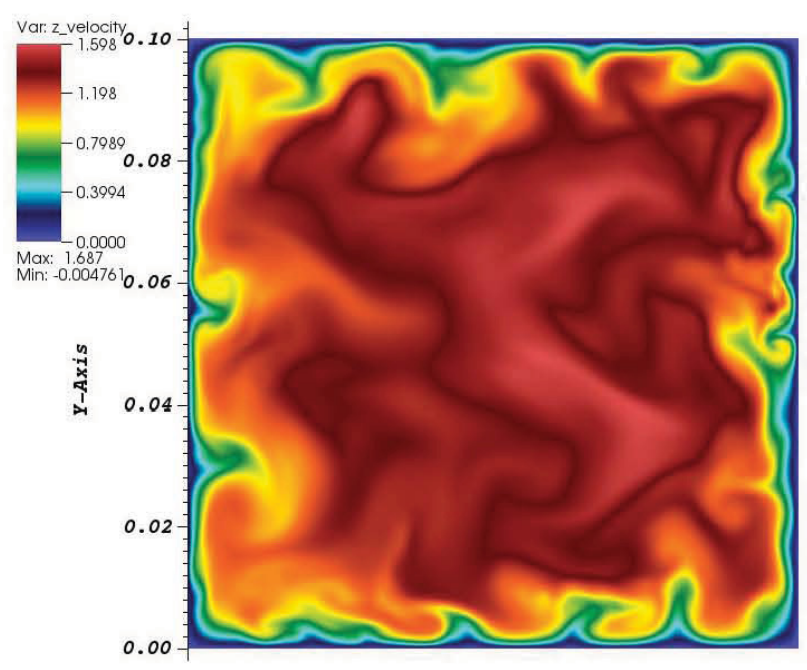

Fine mesh instantaneous velocity

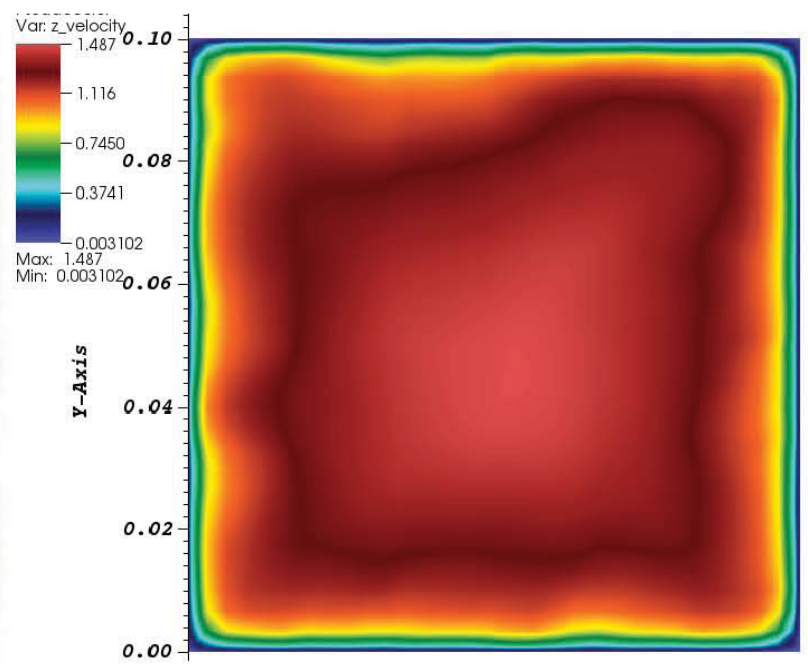

Time and volume-averaged velocity

Figure S-1. Stream-wise velocity contours at 7 seconds.
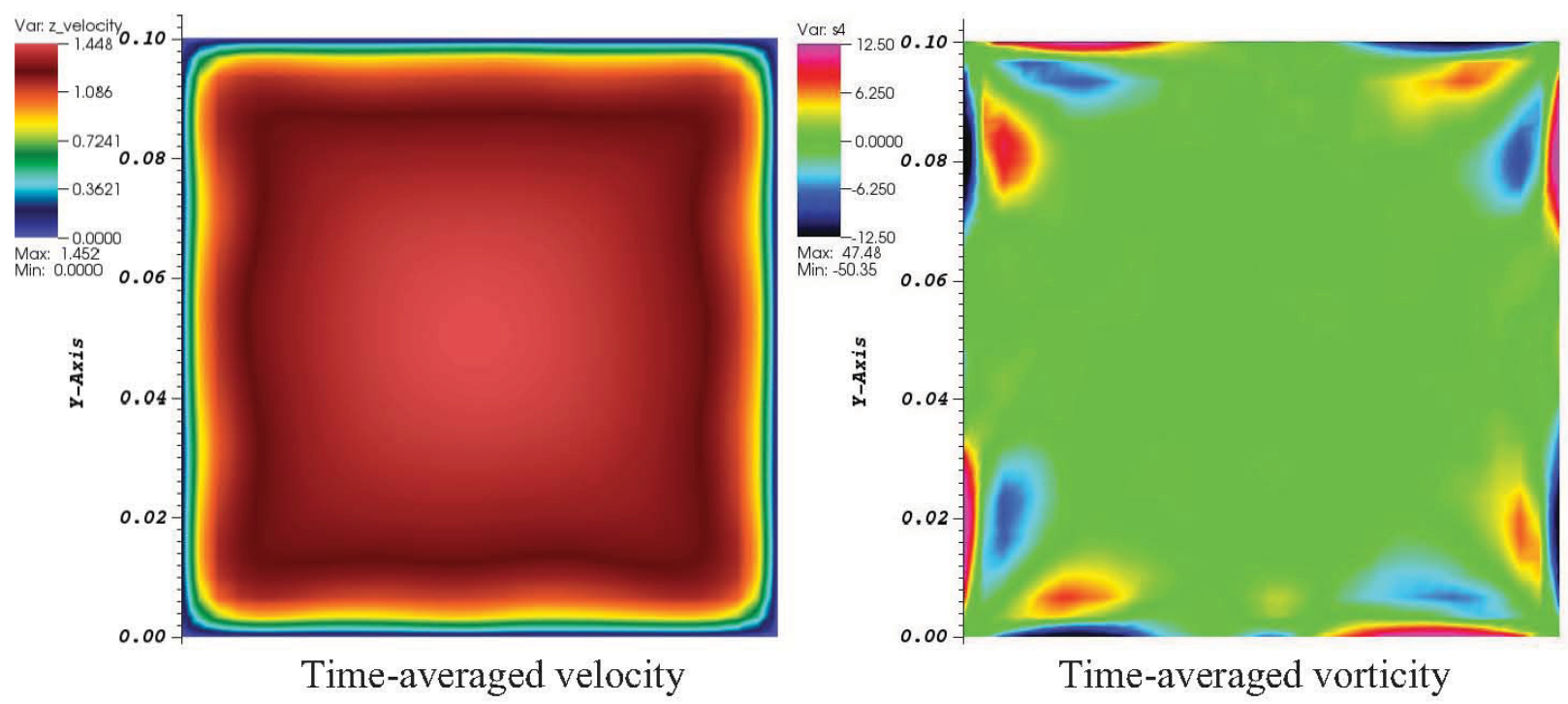

Figure S-2. Stream-wise velocity and vorticity contours on the coarse mesh at 130 seconds. 


\section{Dynamic Multiscale Averaging (DMA) of Turbulent Flow}

\section{Introduction and Background}

One of the most enduring challenges in the field of computer modeling of fluid flow is the challenge of capturing the effects of turbulence on a flow field. Usually, the full detailed effects are not desired, only the average effects on a time scale significantly larger than that of the turbulent fluctuations of the flow field. Initial attempts to model the effects of turbulence were based on the concept of an eddy viscosity, while time-averaging the describing Navier-Stokes equations for stationary flows - turbulent flows with no unsteadiness other than the turbulence. Such equations are called the Reynolds-averaged Navier Stokes or RANS equations, where the averaging is time-averaging for stationary flows and ensemble-averaging for nonstationary flows.

Since the eddy viscosity contains dimensions of length and velocity, modeling efforts focused on proposing prescriptions for the length and velocity scales. The velocity scale was usually based on the turbulent kinetic energy, which has dimensions of velocity squared. Length scales were based on distance from the wall. Eventually, differential transport equations were derived and devised for the turbulent kinetic energy and some other quantity, such as dissipation rate, from which length scales were obtained. Eventually, differential transport equations were derived for the turbulent Reynolds stresses that appear in the RANS equations and solved for each unique stress. Unfortunately, such transport equations contain terms that are even more complex than the Reynolds stresses, such as triple correlations, as compared to the Reynolds stresses, which are double correlations, and must also be modeled. Scores of turbulence models have been developed over the years that are used with the RANS equations; such are often referred to as RANS turbulence models. RANS turbulence models have been successfully used for many industrial applications over the years and are still useful for many flow simulations. Wilcox (1993) provides an excellent overview of RANS turbulence models and their applications.

As computers have increased by orders of magnitude in memory capability and raw speed from early digital computers, new paradigms for computing turbulent flows have been developed. A straightforward approach developed in the third quarter of the $20^{\text {th }}$ century is direct numerical simulation (DNS) of turbulence, where the unsteady Navier Stokes equations are directly solved on finite meshes that are fine enough to capture all of the scales of turbulence, including the Kolmogorov scales where the turbulent motions are dissipated through viscous action. However, it was discovered that as the Reynolds number of the turbulent flow increased, the Kolmogorov 
scales decreased in size, making the computation of large Reynolds number flow very impractical, especially for industrial applications. Nevertheless, advances have been made using DNS, including an increasing array of applications, as compute power has increased, especially the application of parallel computing.

An approach for computing turbulent flow that lies between the RANS approach and DNS is generally called large eddy simulation (LES). Large eddy simulation is based on the notion that the Navier Stokes equations can be filtered, that is, the small scales of motion can be removed such that only the larger scales are directly computed, as in DNS, while the small scales are modeled, usually with simple eddy viscosity models, as in some RANS approaches. The philosophy for using this approach is that the fine scale motions are more isotropic and can easily be modeled, while the larger scale motions are simply computed. Much research has been performed on the LES approach in recent years, while development of new RANS models has tapered off. The model for the small scales is called a sub-grid scale (SGS) model. A large amount of effort has gone into developing better SGS models, as more complex flows seem to require such. Sagaut et al. (2006) provide an excellent overview of LES, DNS and related approaches to turbulence simulation.

One of the LES type of approaches that has been developed by T. J. R. Hughes and colleagues, see Hughes et al. (1998), Sagaut (2006) and Bazilevs et al. (2007), is called variational multiscale methods (VMS). VMS was originally developed for more general applications and has been more recently applied to fluid dynamics. Furthermore, it has evolved from its earlier definition, see Bazilevs (2007). Initially, VMS divided the turbulence scales into three groups, two resolved and a fine unresolved one. More recently, only two groups are employed, a coarse and a fine scale group. The two groups are separated by a projection operator. For spectral methods, this could be applying a wave number cutoff in Fourier space to separate the two scales. The fine scales are then solved approximately using an infinite perturbation series expansion. VMS avoids filtering the describing equations and the attendant problems that comes with such filtering, such as additional terms that arise from non-commutative filter properties. Bazilevs (2007) employs higher order (quadratic and cubic) B-spline basis functions to represent the solutions, declaring that they have better approximation properties than classical higher-order finite elements.

Another approach by Menon and associates (Kemenov and Menon, 2006) is labeled the two level simulation (TLS). In the TLS approach, the velocity field is decomposed into a large-scale (LS) component and a small-scale (SS) component. The decomposition of the primary variable is inserted into the original Navier-Stokes equation, yielding a new set of equations for the LS and the SS variables. The approach undertakes to solve the SS field, but only on lines in each of the three directions, as opposed to volumetric computations. It is shown that this reduces compute time, versus DNS, but introduces approximations as the full 3-D nature of the SS motions is not captured. An example is given for forced isotropic turbulence and compared to DNS. Further work is suggested to improve the method. 
The present work introduces a new approach or paradigm for the computation of turbulence in fluid dynamics. The approach involves multiscale computations that include averaging over space and time. The new approach is called dynamic multiscale averaging (DMA). The describing Navier-Stokes equations are averaged over space and time for multiple scales. Terms appear in the space- and time-averaged equations that represent the effects of averaging and effectively link adjacent scales for momentum and mass conservation. The nature of the approach allows for an unspecified number of scales to be involved in the averaging process. It is expected that the number of scales needed will be problem dependent. The idea is to allow fine-scale calculations to proceed (essentially a DNS initially) until sufficient characterization of the finest resolved scales of the flow field is obtained at the current scale before performing averaging. A running time averaging process is performed during the DNS phase. At an appropriate time, the time-averaged flow field is then averaged spatially. The computation then continues at the new coarser spatial scale and with a longer time step for the averaged flow-field variables until the flow field is again sufficiently characterized and can again undergo averaging. This paradigm continues until the temporal and spatial scales are sufficiently large for the problem being simulated. The computation is akin to an LES after the first set of averaging procedures are performed, except that no filtering has taken place and the finest scales are not modeled, but actually computed. Further temporal and spatial averaging may be comparable to a RANS computation, again without any turbulence modeling. Or, in other words, the SGS model for the LES or the RANS turbulence model is replaced by terms estimated from actual fine-scale calculations. Furthermore, it is envisioned that the averaging processes may need to be reversed, in the case of a non-stationary turbulent flow (where a non-turbulent unsteadiness is also present).

The following sections provide the initial describing equations of fluid dynamics. These equations are then temporally and spatially averaged. Application of the approach using commercial and DOE-NE laboratory codes is then provided.

\section{Equations for Dynamic Multiscale Averaging}

The new paradigm is initially applied to simple incompressible isothermal single phase flow with constant properties and with no body forces. The describing equations in Cartesian coordinates for this flow are given in tensor notation as follows:

conservation of mass:

$$
\frac{\partial u_{j}}{\partial x_{j}}=0
$$

conservation of momentum:

$$
\rho \frac{\partial u_{j}}{\partial t}+\rho u_{k} \frac{\partial u_{j}}{\partial x_{k}}=-\frac{\partial p}{\partial x_{j}}+\mu \frac{\partial^{2} u_{j}}{\partial x_{k} \partial x_{k}}
$$


where repeated indices indicates summation. Equation (2) can be modified by invoking Eqn. (1) to obtain:

conservation of momentum:

$$
\rho \frac{\partial u_{j}}{\partial t}+\rho \frac{\partial u_{k} u_{j}}{\partial x_{k}}=-\frac{\partial p}{\partial x_{j}}+\mu \frac{\partial^{2} u_{j}}{\partial x_{k} \partial x_{k}}
$$

Next, consider turbulent motions that occur on the smallest temporal and spatial scales. Then, average the describing equations over these smallest scales. However, it will still be the case that the averaged equations will be subject to turbulent motions over larger temporal and spatial scales. Before performing a time average, the velocity components and pressure are decomposed into a small scale time mean and fluctuating component as follows:

$$
u_{j}=U_{j}+u_{j}^{\prime} ; \quad p=P+p^{\prime} ; \quad U_{j}=\frac{1}{\Delta T} \int_{t_{o}}^{t_{o}+\Delta T} u_{j} d t=\overline{u_{j}}
$$

where the mean velocity components and pressure are integrated over the smallest time scales. The exact specification of time scale is probably dependent on the Reynolds number of the flow as the smallest spatial scales of the flow are a function of the Reynolds number. In fact, it may be necessary to determine the length of time appropriate to use for the time average here dynamically as the flow characteristics are computed using the original equations, Eqns. (1) and (3). Also, the beginning of the time integration can begin at any point in time. Numerically, this should be after any nonphysical perturbations to the initial flow field have been eliminated. Performing the time average on Eqns. (1) and (3) yields equations that look like typical Reynolds averaged Navier-Stokes equations, except that the length of the time applied to the time integral is shortened to include only the small time scales. One obtains:

$$
\frac{\partial U_{j}}{\partial x_{j}}=0
$$

and

$$
\rho \frac{\partial U_{j}}{\partial t}+\rho \frac{\partial U_{k} U_{j}}{\partial x_{k}}+\rho \frac{\partial \overline{u_{k}^{\prime} u_{j}^{\prime}}}{\partial x_{k}}=-\frac{\partial P}{\partial x_{j}}+\mu \frac{\partial^{2} U_{j}}{\partial x_{k} \partial x_{k}}
$$

where the order of integration and differentiation have been interchanged, the overbar indicating the time average, and the cross-terms involving products of mean and fluctuating components have been rendered zero by construction. Because the equations have been averaged only over the smallest time scales, it is expected that the mean quantities, e.g. $U_{j}$ and $P$, will still vary in time over larger time scales.

The small-scale time-averaged describing equations are now spatially averaged over the smallest spatial scales. Again, a decomposition is applied to the now short-time averaged quantities:

$$
U_{j}=\left\langle U_{j}\right\rangle+U_{j}^{\Delta} ; \quad P=\langle P\rangle+P^{\Delta} ; \quad\left\langle U_{j}\right\rangle=\frac{1}{V} \int U_{j} d V
$$


The deviation $U_{j}^{\Delta}$ from the volumetric average $\left\langle U_{j}\right\rangle$ represents the deviation of the time mean at a point, or after discretization, of a small scale mesh cell, from the volumetric average on a larger scale or coarser mesh cell. Through this operation, a new coarser mesh is defined for time and volumetric averaged quantities. However, new terms have appeared in the time and volumetric averaged equation that didn't appear in the fine scale equations. The time and spatially averaged describing equations are:

$$
\frac{\partial\left\langle U_{j}\right\rangle}{\partial x_{j}}=0
$$

and

$$
\rho \frac{\partial\left\langle U_{j}\right\rangle}{\partial t}+\rho \frac{\partial\left\langle U_{k}\right\rangle\left\langle U_{j}\right\rangle}{\partial x_{k}}+\rho \frac{\partial\left\langle U_{k}^{\Delta} U_{j}^{\Delta}\right\rangle}{\partial x_{k}}+\rho \frac{\partial\left\langle\overline{\left.u_{k}^{\prime} u_{j}^{\prime}\right\rangle}\right.}{\partial x_{k}}=-\frac{\partial\langle P\rangle}{\partial x_{j}}+\mu \frac{\partial^{2}\left\langle U_{j}\right\rangle}{\partial x_{k} \partial x_{k}}
$$

where the differentiation and volume integration have been interchanged. As can be seen in Eqn. (9), there are two terms that have appeared that represent connections between the finer scale and the coarser scale. One is the volume averaged fine-scale turbulent fluctuations and one is the volumetric averaged deviation of time mean velocity components from the coarse average. The time and volume averaged momentum equations are still unsteady in terms of larger scale motions. Hence, it is anticipated to use a larger time step for the coarser equations.

It is envisioned that the above processes of computing for a time, then performing time and volume averaging can be repeated to reach larger and larger scales, both in time and space. For example, upon time averaging the primary quantity $\left\langle U_{j}\right\rangle$ from Eqn. (9) yields $\overline{\left\langle U_{j}\right\rangle}$, or the time average of the volume and time averaged velocity. However, the number of levels that would be appropriate is likely problem dependent. However, the newly generated terms provide coupling between adjacent scales. It is also envisioned that the newly created terms coupling lower scales to the current scales would not change significantly in time; thus they can be treated as source terms for the larger scales. The averaged quantities, such as $\left\langle U_{j}\right\rangle$ would be those that would be computed at the larger scales, until the largest scales needed or desired are reached. That is, the effects of the finer scales are computed directly using appropriate time and spatial scales, until representative fields at the scale in question are computed before time and volume averaging are applied. Then the sizes of the time and volume scales are increased using representative couplings between scales that have actually been computed. Hence, there is no modeling required. Based on the above averaging processes, the present approach to computing a turbulent flow field is called "dynamic multiscale averaging" or DMA. It is further envisioned that should disturbances to the flow field be encountered after the starting time of the turbulent field, which would affect the fine scale structure of the evolving field, that the averaging process might be reversed to return to finer scales to capture the desired effects.

In summary, a flow field domain is discretized to a mesh fine enough to allow accurate DNS to proceed. After an appropriate length of time, which would characterize eddy motions at the finest scales, the flow field is temporally and spatially averaged, creating new terms in the 
resulting equations, whose values are computed from the fine mesh flow field. The computations are then recommenced on a coarser mesh, defined by the spatial averaging, at a larger time scale to capture the larger scales. By performing this process, perhaps multiple times, eventually the flow field will be similar to a RANS calculation, except that the coupling correlations derived from the averaging processes will have been computed, rather than modeled. Certainly, it is the case that a fully temporally-developed DNS calculation can be averaged to obtain a RANS-like flow field with accompanying averaged values for the Reynolds stresses. In its least accelerated form, DMA would be exactly such a process. However, if the fine scales can be computed for a short time, representative of the time scales therein, and so on up through the scales, then the computation of the final flow field can be accelerated, perhaps greatly so such that this method, DMA, can be applied to typical industrial applications in a time frame comparable to that of a RANS calculation, and without traditional turbulence models that characterize the RANS approach to computing turbulent flow.

\section{Application of Dynamic Multiscale Averaging - DMA}

The objective of the present study is to apply dynamic multiscale averaging - DMA - to incompressible, constant property turbulent flow in a simple geometry to investigate its effectiveness and practicality. The geometry selected for performing the study is a segment of a square duct. DNS calculations have been performed for such a square duct geometry, for a relatively small Reynolds number by Raiesi et al. (2011) and Pinelli et al. (2010). The Reynolds stresses in a square duct are known to produce relatively weak secondary flow (in the cross section), making this an interesting flow case to see if such secondary flows can be generated using DMA.

The square duct chosen is similar to that of Raiesi et al. (2011) and Pinelli et al. (2010) with a cross section of $0.1 \mathrm{~m}$ by $0.1 \mathrm{~m}$ and a length of $0.6 \mathrm{~m}$. Constant fluid properties of 1.18415 $\mathrm{kg} / \mathrm{m} 3$ for density and $1.85508 \times 10^{-5} \mathrm{~Pa}-\mathrm{sec}$ for dynamic viscosity are used. Two CFD codes have been employed in the present effort: a commercial CFD code (STARCCM+ version 6.06.011) and the spectral element code Nek5000. Initially, the DNS flow was set up in STARCCM+ with a specified pressure jump from the inlet to the outlet of $-0.15 \mathrm{~Pa}$ and with periodic boundaries between the inlet and outlet. This produces a volumetric flow rate of $0.011585 \mathrm{~m}^{3} / \mathrm{sec}$; the computed flow rate is then used for the Nek5000 code to produce a similar flow. The Nek5000 flow problem is also set to have periodic boundaries from the inlet to the outlet. These parameters yield a Reynolds number of 3697, based on half the width of the cross section, comparable to the Reynolds number of 3535 used in Pinelli et al. (2010). A time step size of $5.0 \times 10^{-4} \mathrm{sec}$ is used for both codes for the initial stage of the DMA study.

The first stage of the proposed DMA is to perform a proper but brief DNS calculation. To achieve turbulent flow, a nonphysical asymmetric initial flow condition is specified. A grid of $200 \times 200 \times 400$ cells was constructed for the STARCCM+ calculation. The grid is finer near the wall. Figure 1 illustrates a cross-sectional view of the velocity magnitude at the axial half-

\footnotetext{
${ }^{a}$ http://nek5000.mcs.anl.gov/index.php/Main_Page
} 
way point of the square duct segment for the STARCCM+ calculation at compute time of 10.1 seconds; it required about 5 seconds for turbulence to appear in the DNS. It can be seen that turbulent motions are apparent in the snapshot view of the flow field.

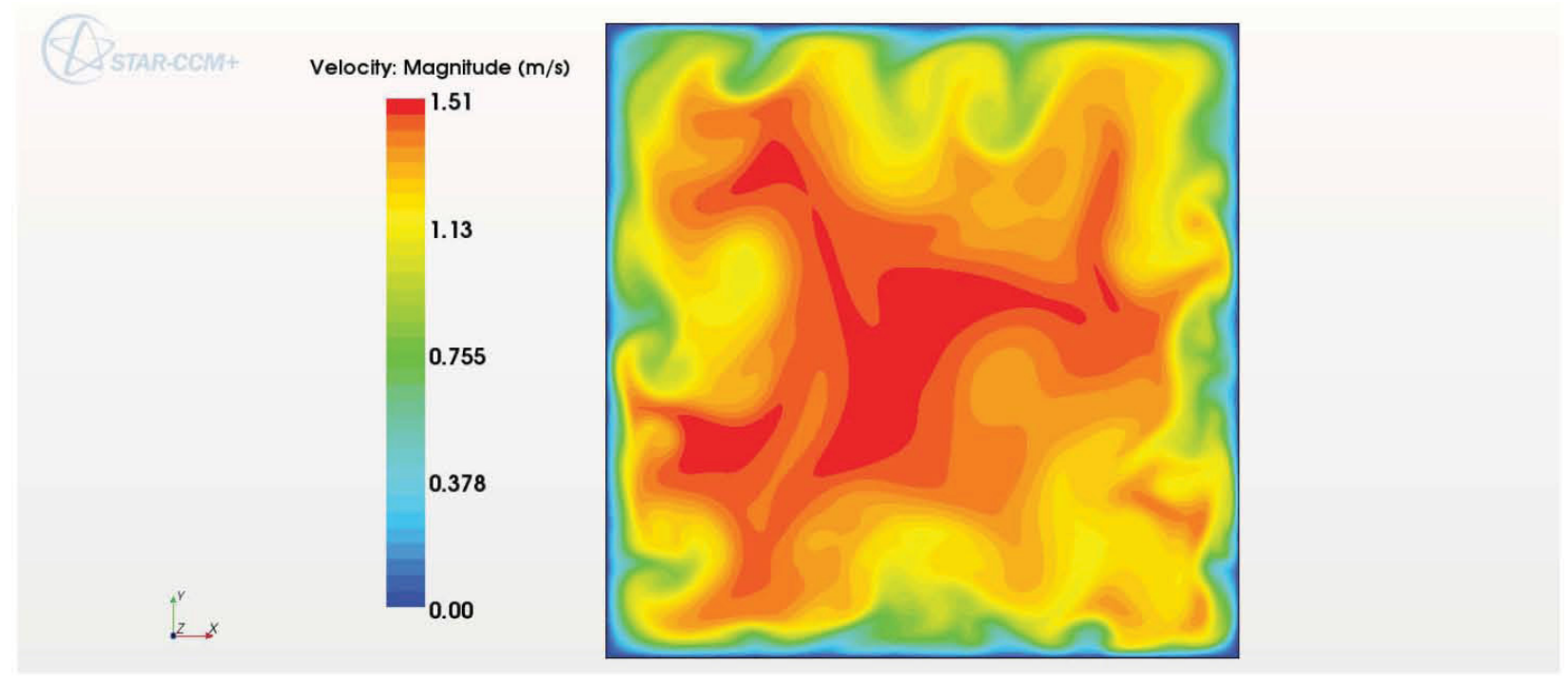

Figure 1. Velocity magnitude for the STARCCM+ DNS calculation at $10.1 \mathrm{sec}$.

Figure 2 illustrates the velocity magnitude of the flow at the duct center plane computed using Nek5000 at 7 seconds of compute time. The grid is a uniform spectral element grid of $24 \times 24 \times$ 32 elements in the $\mathrm{x}, \mathrm{y}$ and $\mathrm{z}$ directions, the main flow being in the $\mathrm{z}$-direction. Seventh order spectral elements are used for the simulation; therefore each spectral element is defined by an $8 \mathrm{x}$ $8 \times 8$ set of points, with velocity component and pressure values computed for each point. Hence, the spectral grid is effectively defined by $169 \times 169 \times 225$ points; the boundary points for each spectral element coincide with boundary points for adjacent elements. An asymmetric initial condition is also specified for the Nek5000 computation. It can be seen that turbulent motions are present in the flow field. The DNS calculation required $9.9 \mathrm{hrs}$ per second of simulation time using 24 parallel cpus.
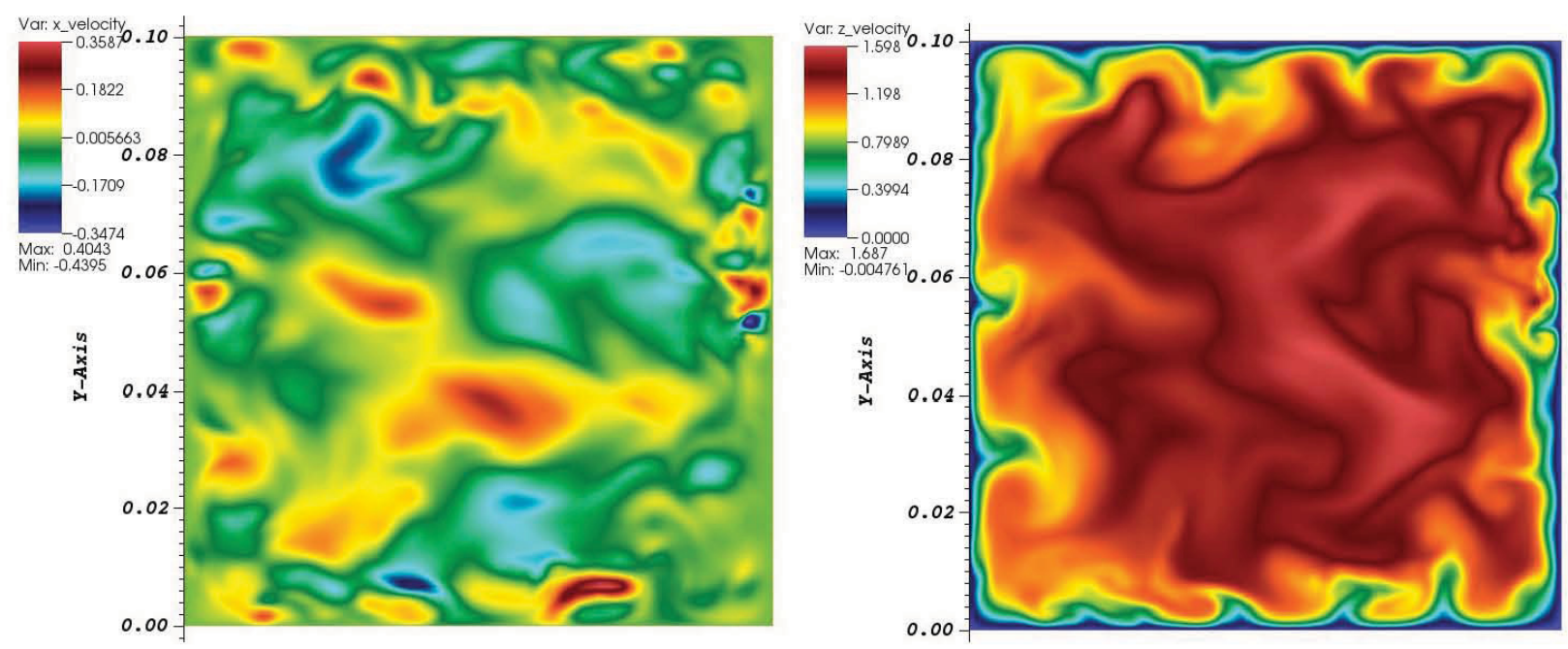

Figure 2. Instantaneous $u$ and $w$ velocities for Nek5000 DNS at 7 seconds. 
The second stage of DMA requires first time and then volume averaging of the flow field, including creating correlations $\left\langle\overline{u_{\imath}^{\prime} u_{j}^{\prime}}\right\rangle$ and $\left\langle U_{i}^{\Delta} U_{j}^{\Delta}\right\rangle$. Both STARCCM+ and Nek5000 have builtin running time-averaging available which yields the time mean velocity components and pressure as well as variances and covariances of products of the velocity components, from which the turbulent or Reynolds stresses can be computed. Figure 3 illustrates contours of time mean stream-wise velocity for the STARCCM + calculation at 10.1 seconds. It can be seen that it is not yet symmetric about the center lines; eventually, the mean stream-wise velocity should become symmetric about lines drawn vertically and horizontally through the duct center and also about diagonal lines drawn between opposite corners.

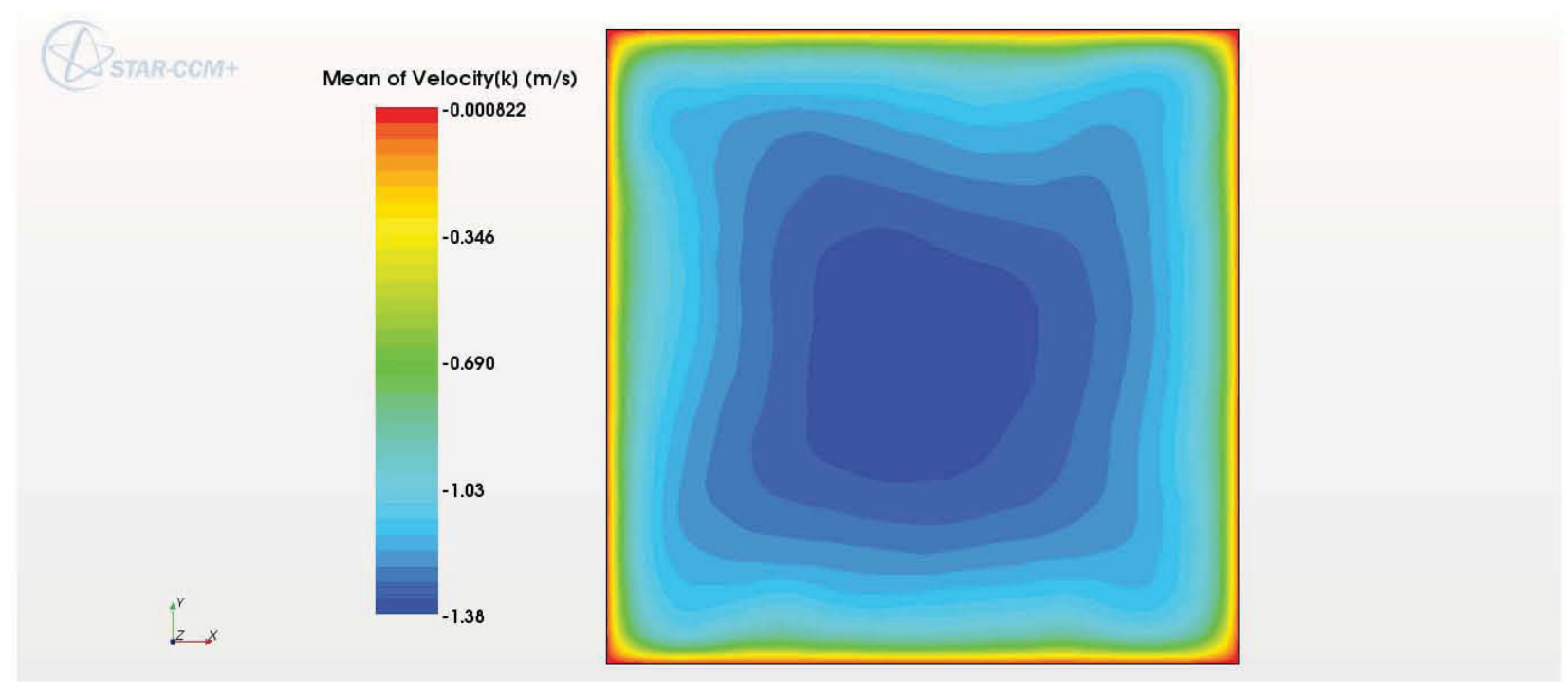

Figure 3. Time-average $W$ velocity for STARCCM + at $10.1 \mathrm{sec}$.

Figure 4 illustrates contours of the time-averaged $U$ and $W$ velocity components, averaged from 5 to 7 seconds for the Nek5000 calculations. It can be seen that there is rough symmetry about a horizontal line through the centre for $U$ and about both horizontal and vertical lines through the center for $W$. The symmetry would be much better had the time-averaging been performed over a longer period. After performing time-averaging, it is desired to perform local volumetricaveraging as described in Sec. 2. A volumetric averaging procedure was developed and coded into a separate utility code to perform volumetric averaging on time averaged quantities from the Nek5000 calculation. Figure 5 illustrates contours for the time-averaged velocity component $V$ on the fine $24 \times 24 \times 32$ grid and the time and volume-averaged $\langle V\rangle$ on the coarser $6 \times 6 \times 8$ grid, with both grids shown. It can be seen that the contours are quite similar on the two grids. Again, there is very rough symmetry about a vertical line through the center for $V$ in this plot. Note that the collocation points, or Gauss-Lobatto-Legendre points, for the spectral elements (points where the mesh lines intersect) are spaced more finely near the element edges.

Figure 6 illustrates contours for the time and volume-averaged velocities $\langle U\rangle$ and $\langle W\rangle$ on the coarser mesh. Comparing these illustrations with those of Fig. 4, the time-averaged components on the fine grid, it appears that the contours are very similar before and after volume averaging. 

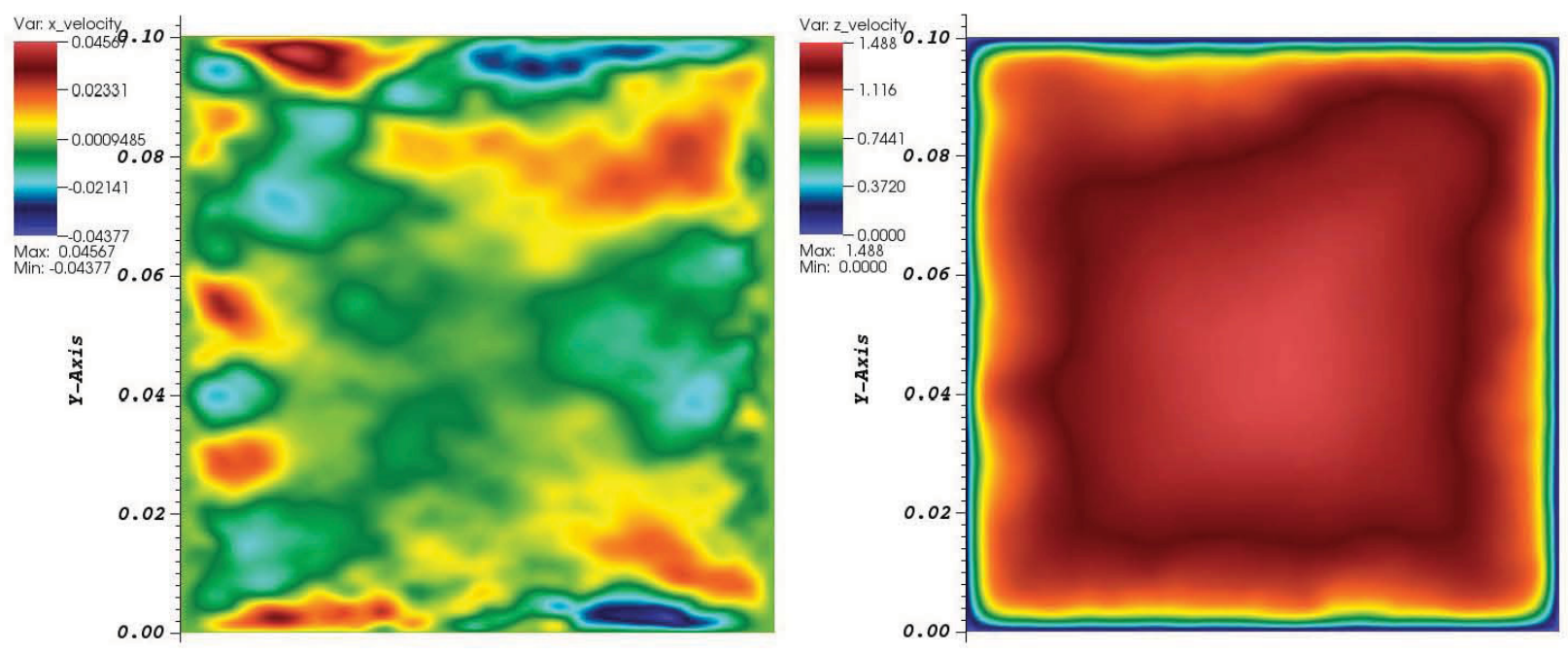

Figure 4. Time-averaged $U$ and $W$ velocities from $5-7$ seconds for Nek5000.
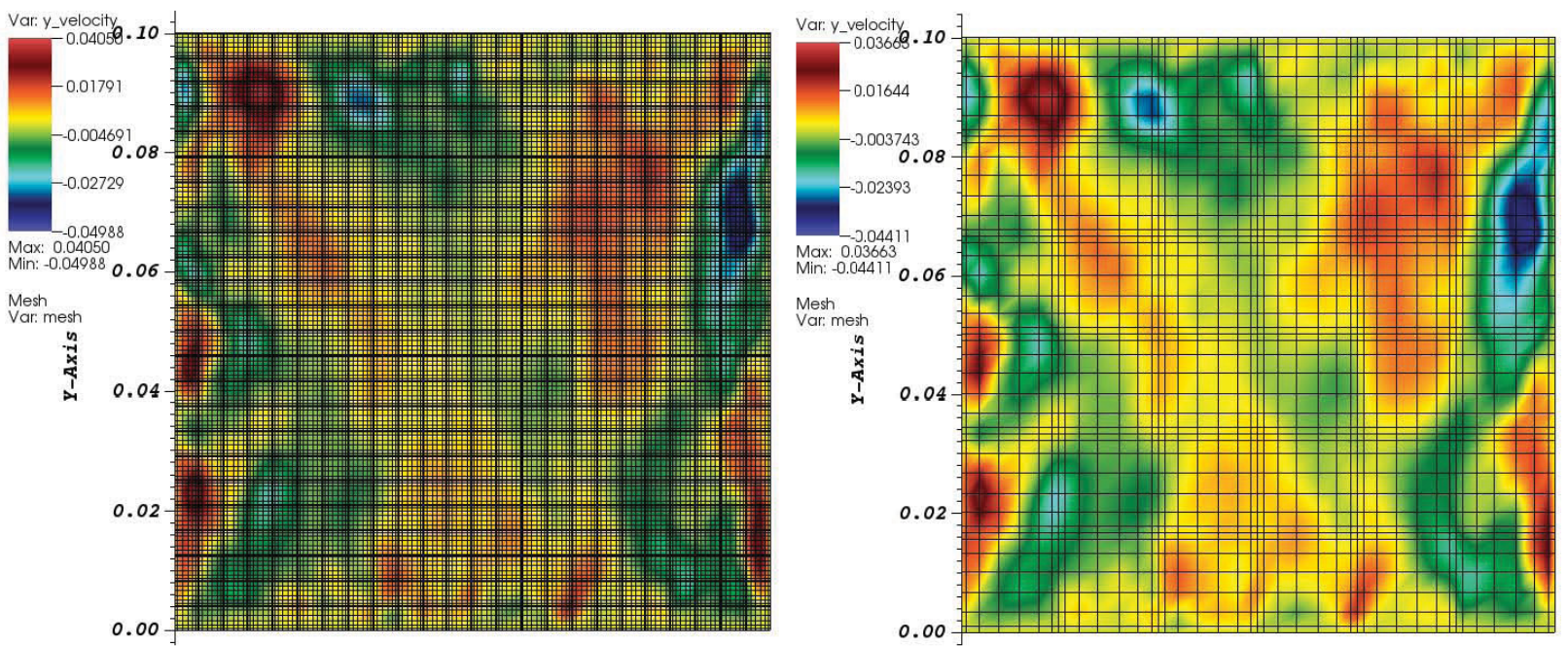

Figure 5. Time-averaged $V$ on fine grid and time \& volume-averaged $\langle V\rangle$ on coarse grid.
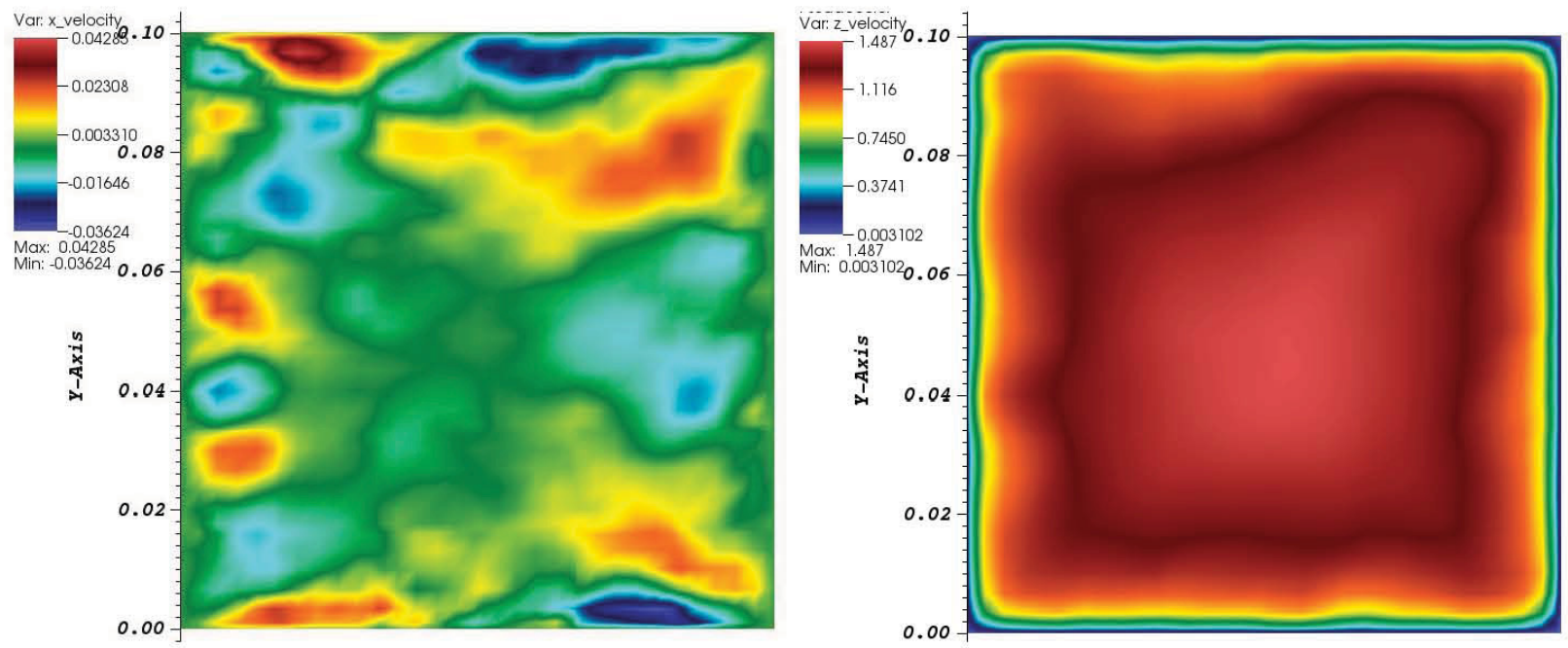

Figure 6. Time and volume-averaged $\langle U\rangle$ and $\langle W\rangle$ at 7 seconds for Nek5000. 
Figure 7 illustrates the time and volume-averaged variances and co-variances $\left\langle U^{\Delta} U^{\Delta}\right\rangle,\left\langle W^{\Delta} W^{\Delta}\right\rangle$, $\left\langle U^{\Delta} V^{\Delta}\right\rangle$, and $\left\langle U^{\Delta} W^{\Delta}\right\rangle$; see Eqn. (9). The other variance $\left\langle V^{\Delta} V^{\Delta}\right\rangle$ is similar to $\left\langle U^{\Delta} U^{\Delta}\right\rangle$ and the other co-variance $\left\langle V^{\Delta} W^{\Delta}\right\rangle$ is similar to $\left\langle U^{\Delta} W^{\Delta}\right\rangle$ (when rotated 90 degrees), as expected. These correlations are related to the deviation of the fine-grid time mean from the time and volume means on the coarse grid. It can be seen that the peak values for these correlations occur near the walls. Also note that the cross-stream variance $\left\langle U^{\Delta} U^{\Delta}\right\rangle$ is two orders of magnitude smaller than the stream-wise correlation $\left\langle W^{\Delta} W^{\Delta}\right\rangle$ and that the cross-stream co-variance $\left\langle U^{\Delta} V^{\Delta}\right\rangle$ is an order of magnitude smaller than the co-variance $\left\langle U^{\Delta} W^{\Delta}\right\rangle$.
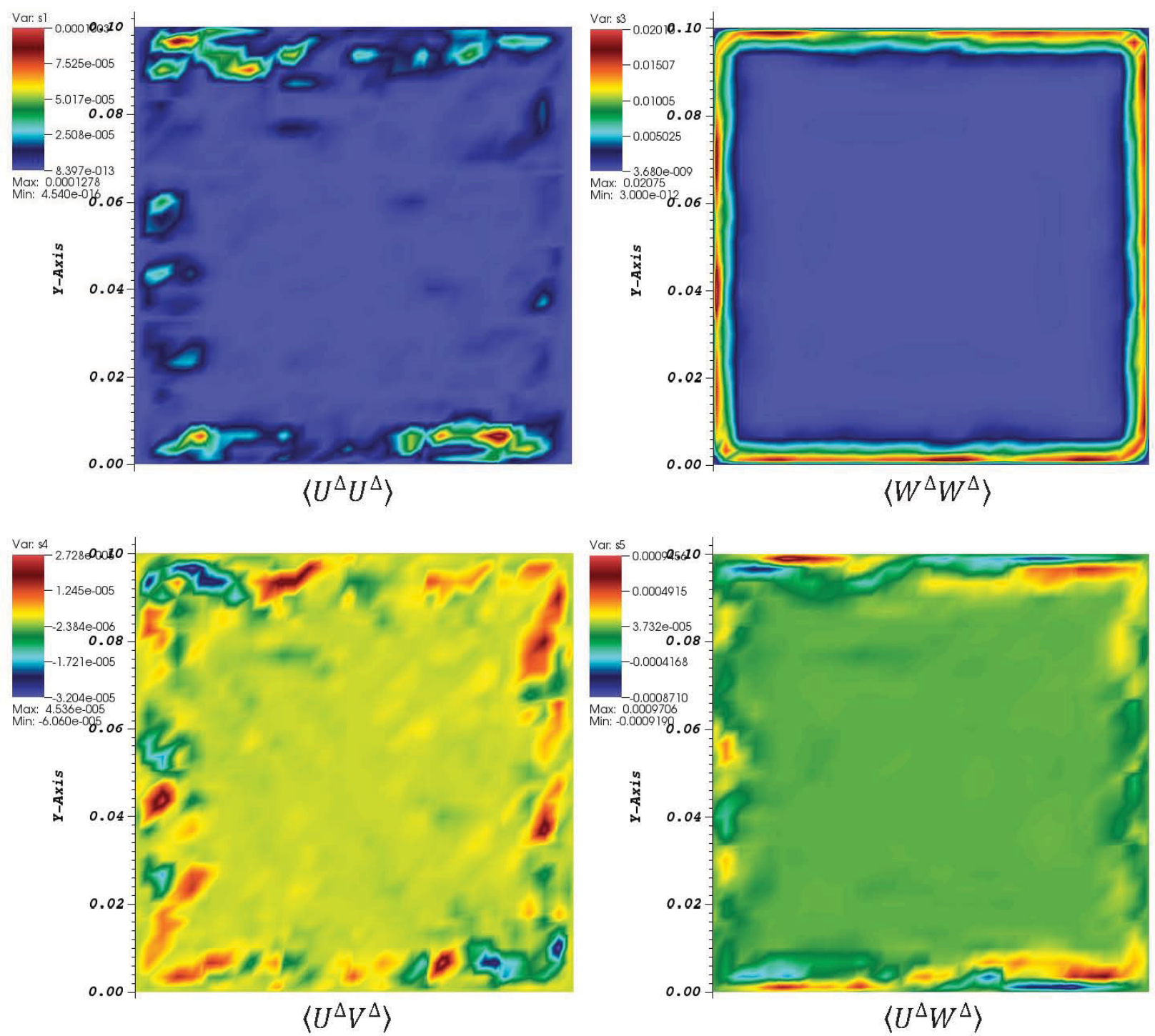

Figure 7. Time and volume-averaged variances and co-variances of the deviation of fine-grid volume values from the coarse-grid volume average.

Figure 8 illustrates the time and volume-averaged turbulence-related variances and covariances $\left\langle\overline{u^{\prime} u^{\prime}}\right\rangle,\left\langle\overline{w^{\prime} w^{\prime}}\right\rangle,\left\langle\overline{u^{\prime} v^{\prime}}\right\rangle$ and $\left\langle\overline{u^{\prime} w^{\prime}}\right\rangle$, see Eqn. (9). The other variance $\left\langle\overline{v^{\prime} v^{\prime}}\right\rangle$ is similar to $\left\langle\overline{u^{\prime} u^{\prime}}\right\rangle$ and the other co-variance $\left\langle\overline{v^{\prime} w^{\prime}}\right\rangle$ is similar to $\left\langle\overline{u^{\prime} w^{\prime}}\right\rangle$ (when rotated 90 degrees), as expected. There is clear, albeit rough, symmetry for both $\left\langle\overline{u^{\prime} u^{\prime}}\right\rangle$ and $\left\langle\overline{w^{\prime} w^{\prime}}\right\rangle$ across horizontal and vertical 
lines through the duct center. The co-variance $\left\langle\overline{w^{\prime} w^{\prime}}\right\rangle$ also shows rough symmetry about diagonal lines between opposite corners, as it should. The co-variance $\left\langle\overline{u^{\prime} v^{\prime}}\right\rangle$ exhibits rough symmetry about the diagonal lines, while the co-variance $\left\langle\overline{u^{\prime} w^{\prime}}\right\rangle$ shows rough symmetry about vertical and horizontal lines drawn through the duct center. For the turbulence correlations, it is apparent that the cross-stream component $\left\langle\overline{u^{\prime} u^{\prime}}\right\rangle$ is only about a factor of four smaller than the stream-wise component $\left\langle\overline{w^{\prime} w^{\prime}}\right\rangle$, while the cross-stream co-variance term $\left\langle\overline{u^{\prime} v^{\prime}}\right\rangle$ is also only about a factor of four less than the co-variance $\left\langle\overline{u^{\prime} w^{\prime}}\right\rangle$. Thus, the turbulence correlations are much larger than the correlations related to volume averaging and there is much less variation in peak magnitude between turbulent co-variances than between those related to volume averaging.
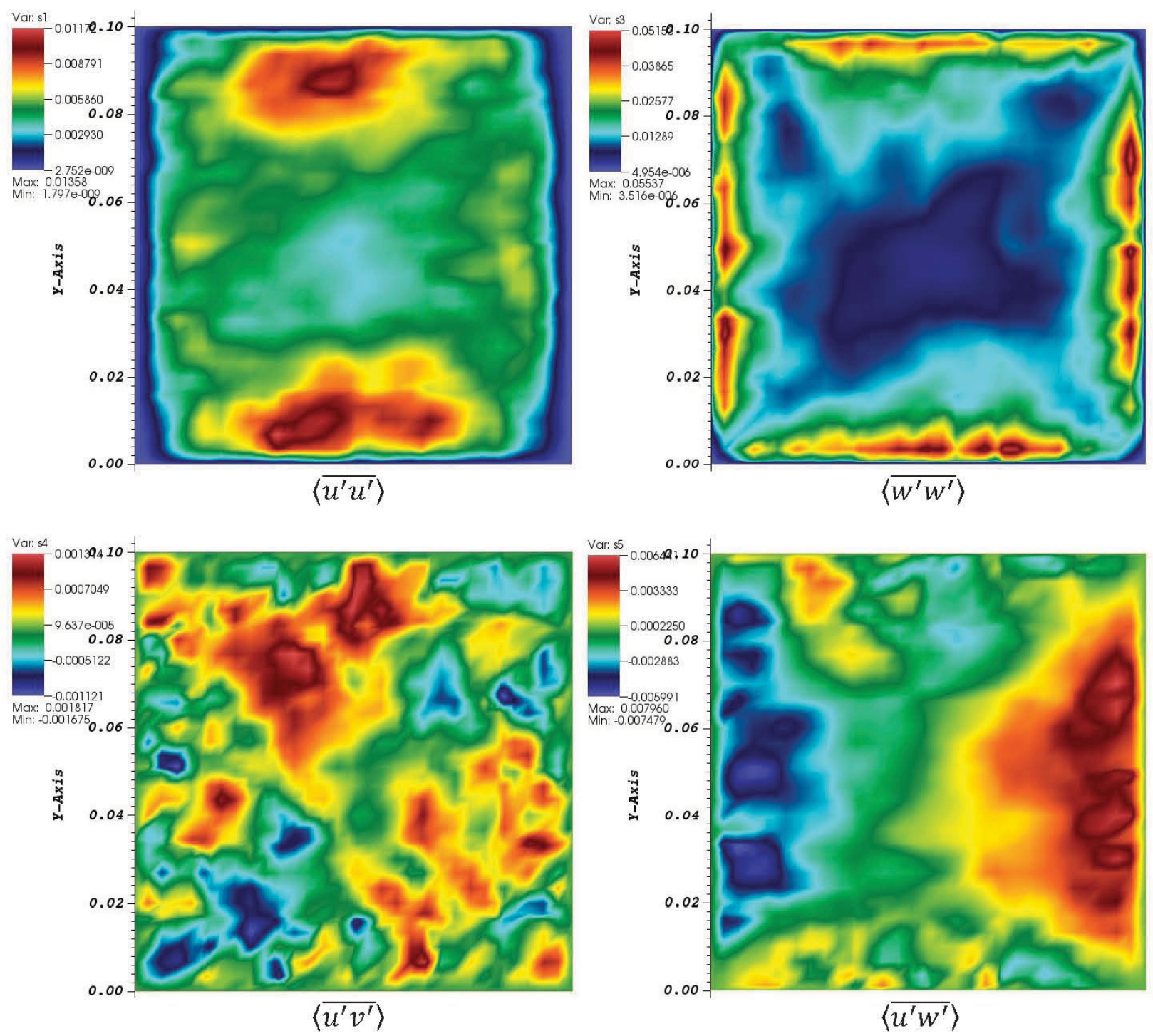

Figure 8. Time and volume-averaged variances and co-variances of that are related to the turbulent fluctuations.

The third stage of DMA is to start the calculation on the coarser grid using the time and volume averaged quantities $\left\langle U_{i}\right\rangle$ and $\langle P\rangle$ as the primary variables of the computation with the terms involving the newly generated correlations $\left\langle\overline{u_{i}^{\prime} u_{j}^{\prime}}\right\rangle$ and $\left\langle U_{i}^{\Delta} U_{j}^{\Delta}\right\rangle$ added as constant source terms. Note that it is the gradients of these correlations that appear in the describing equations, Eqn. (9). 
Also note that the code Nek5000 can simply be invoked as if the original primitive variables used for the DNS were being used. Of course, the terms involving the new correlations are added as source terms. Interestingly, the computations of the third stage behave as if an LES were being computed. After only a short time, the time and volume-averaged fields of Figs. 5 and 6 resume a fluctuating field, as for the initial DNS calculation, albeit on the coarser grid. Figure 9 illustrates the field for $\langle U\rangle$ and $\langle W\rangle$ at 0.5 seconds beyond the initiation of the third stage of DMA. Note that the computations required about $139 \mathrm{sec}$. per $1 \mathrm{sec}$. of simulation time for 12 cpus. Comparing this to the DNS case, and assuming that the parallel scaling is perfect, this decrease in compute time for the same simulation time is a factor of about 510. Of course, this is only valuable if the final results of the coarse-scale calculation are reasonably accurate compared to the DNS or to reality.
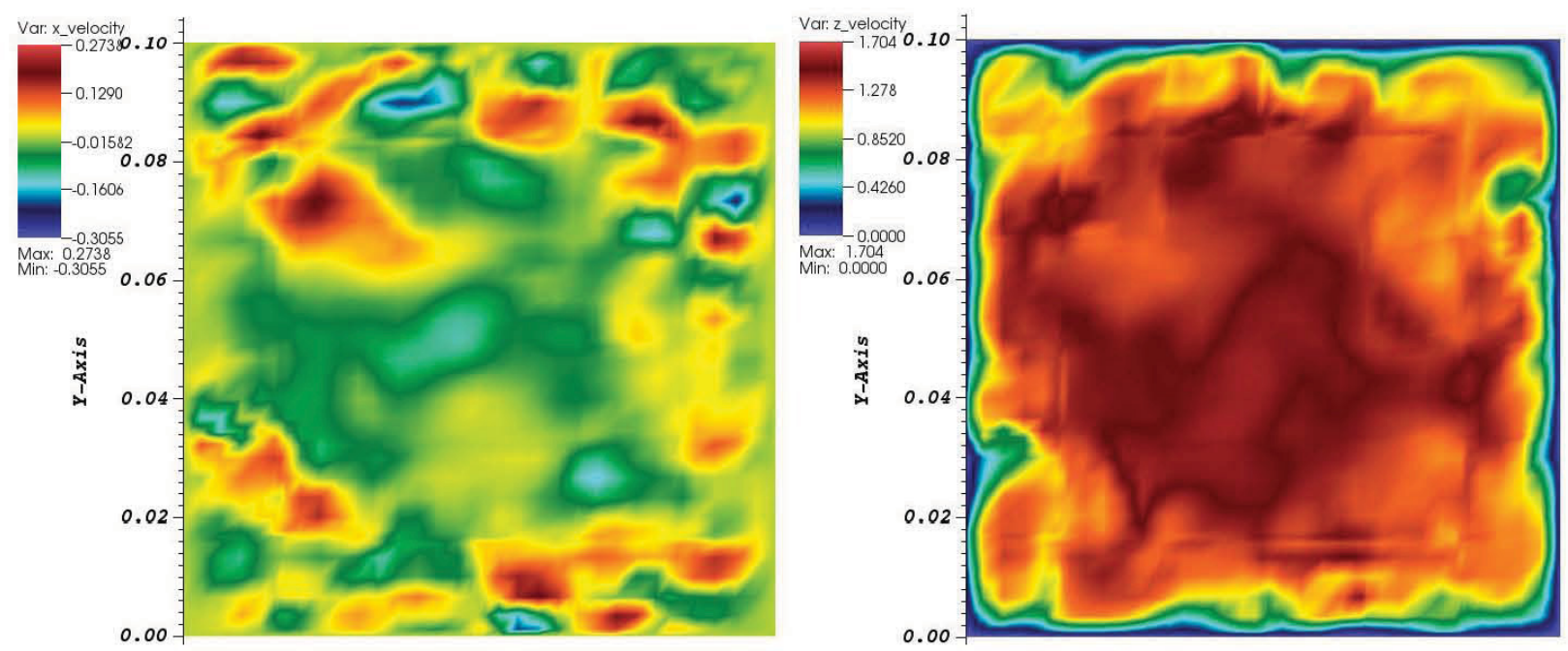

Figure 9. Time evolution of time and volume-averaged velocity components $\langle V\rangle$ and $\langle W\rangle$ after $0.5 \mathrm{sec}$ of the initiation of computation on coarser grid.

Computations have been carried out to 130 seconds (including initial DNS simulation time) for the third stage of DMA. During this calculation, a running time average has been kept of the primary variables and of the vorticity. To visualize the development of the present flow field, the stream-wise vorticity component is plotted for four times, including 55, 80, 105 and 130 seconds in Figure 10. As can be seen, the contours across vertical, horizontal and diagonal lines through the center improve with simulation time and are quite close to being symmetric. Furthermore, the vorticity contours shown are very similar to those reported by Raiesi et al. (2011) for their DNS in a square duct. The vorticity patterns exhibited in Fig. 10 are indicative of secondary flow where the flow in the cross-sectional plane moves towards the corners, turns and flows towards the centers of the sides and then back towards the duct center. Such a flow pattern is well-known and is a result of anisotropic turbulent motions. 

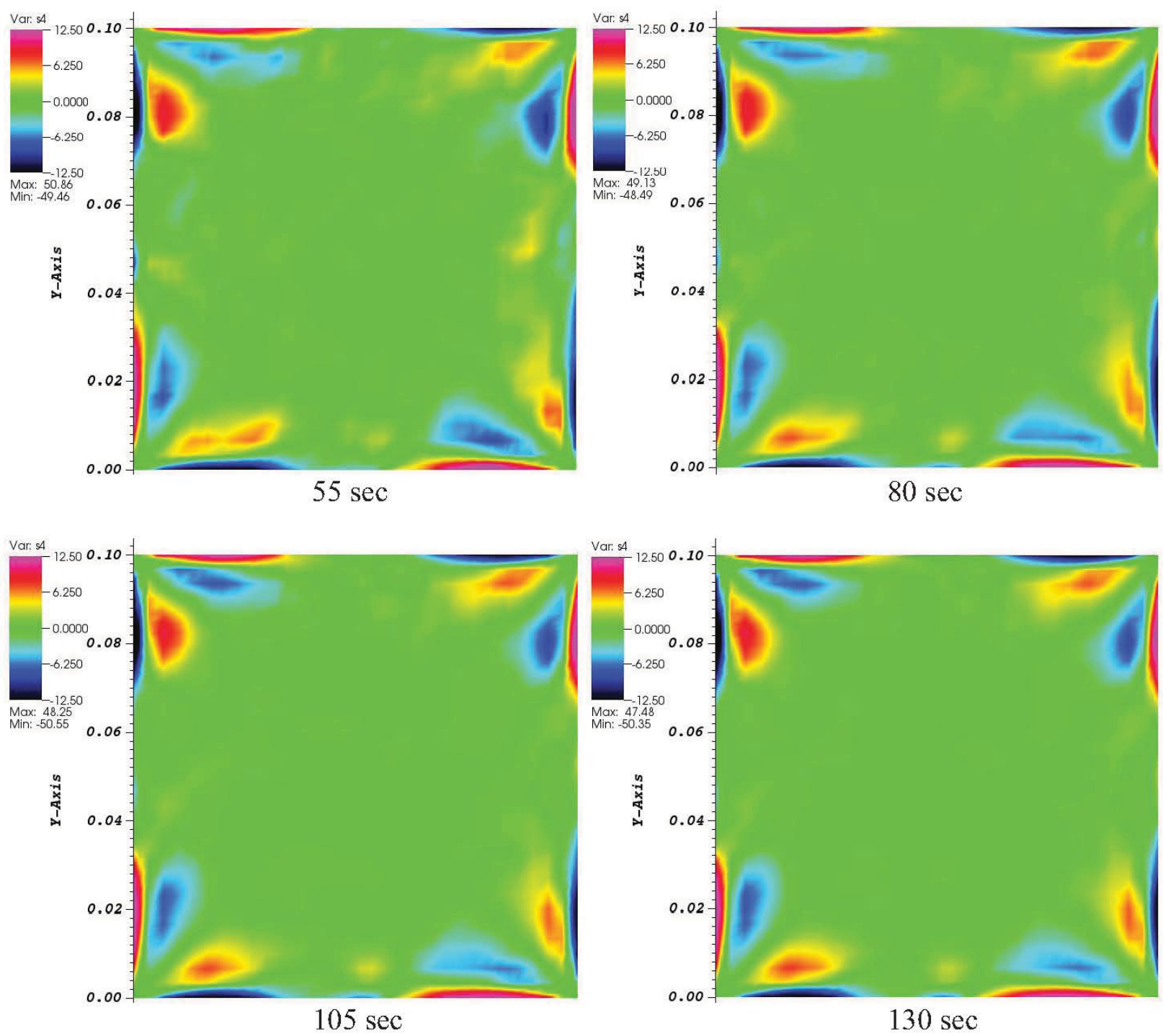

Figure 10. Time-averaged, time and volume-averaged stream-wise vorticity component contours on the coarser grid at various simulation times.

Figure 11 provides contour plots of the time-averaged, time and volume-averaged velocity components $\overline{\langle U\rangle}, \overline{\langle V\rangle}$, and $\overline{\langle W\rangle}$ and the pressure $\overline{\langle P\rangle}$. That is, the plots show the time mean of the flow field on the coarse grid. The results for $\overline{\langle U\rangle}$ and $\overline{\langle V\rangle}$ are both fairly symmetric about horizontal and vertical lines through the center. They should also be very similar upon rotation of the plot by 90 degrees, which they are. The results for and $\overline{\langle W\rangle}$ and $\overline{\langle P\rangle}$ are seen to be quite symmetric about vertical, horizontal and diagonal lines drawn through the center, as they should be for a temporally fully-developed DNS. The contours for $\overline{\langle W\rangle}$ closely resemble contours for the stream-wise velocity illustrated in Raiesi et al. (2011) for their DNS calculations. The present calculations can be seen as somewhat equivalent to an LES, except that the sub-grid scale model is not a model, but a field based on a short time DNS computation, providing an estimate, based on first principles, of the coupling between the fine and coarse scales of the flow field. It is interesting to note that even though the correlations $\left\langle\overline{u_{i}^{\prime} u_{j}^{\prime}}\right\rangle$ and $\left\langle U_{i}^{\Delta} U_{j}^{\Delta}\right\rangle$ (which are being used for the coarse grid calculations as constant source terms) are only roughly symmetric and 
therefore approximate, as shown in Figs. 7 and 8, the eventual flow field has become quite symmetric, as seen in Figs. 10 and 11. One may then conclude that the DMA approach shows great potential to obtain the important effects of the turbulent flow field.
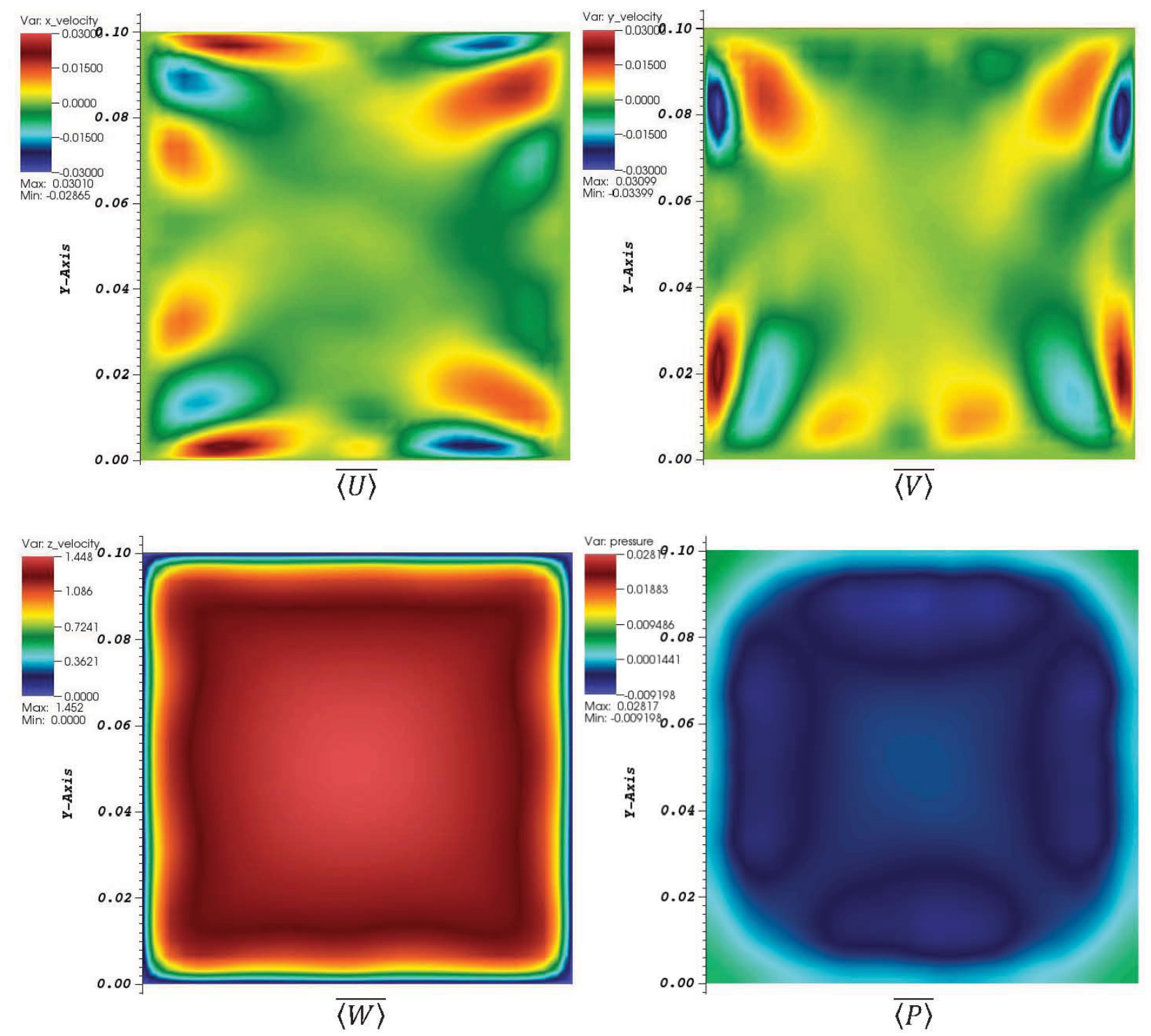

Figure 11. Time-averaged, time and volume-averaged flow field components on the coarser grid at 130 seconds of simulation time.

\section{Summary and Recommendations}

A new approach called dynamic multiscale averaging (DMA) for computing the effects of turbulent flow is described. The new method encompasses multiple applications of temporal and spatial averaging, that is, multiscale operations. Initially, a direct numerical simulation (DNS) is performed for a relatively short time; it is envisioned that this short time should be long enough to capture several fluctuating time periods of the smallest scales. The flow field variables are subject to running time averaging during the DNS. After the relatively short time, the time- 
averaged variables are volume averaged onto a coarser grid. Both time and volume averaging of the describing equations generate correlations in the averaged equations. These correlations are computed from the flow field and added as source terms to the computation on the next coarser mesh. They represent coupling between the two adjacent scales. Since they are computed directly from first principles, there is no modeling involved. However, there is approximation involved in the coupling correlations as the flow field has been computed for only a relatively short time. After the time and spatial averaging operations are applied at a given stage, new computations are performed on the next coarser mesh using a larger time step. The process continues until the coarsest scale needed is reached. New correlations are created for each averaging procedure. The number of averaging operations needed is expected to be problem dependent.

The new DMA approach is applied to a relatively low Reynolds number flow in a square duct segment. Results for each stage of the method are provided for various field variables and their temporal and volume averaging. The DNS computation is initiated using a nonphysical asymmetric initial condition. After five seconds of simulation time, running time averages are initiated and performed for another two seconds. The time-averaged variables are then volume averaged onto a coarser mesh. The new coupling correlations are also computed. These correlations are assumed to be constant for succeeding computations and are added as source terms. Calculations are then performed using the time and volume-averaged field variables on the coarser grid. The coarser grid is essentially created using a mesh that has been reduced in each coordinate by a factor of four. The time step is increased by a factor of five. The compute time for a given time interval on the coarse mesh is about $1 / 500$ the corresponding time required on the fine mesh. Results for a final time of 130 seconds are presented. Time-averaged streamwise vorticity contours appear to be very similar to a full DNS for a similar flow reported in the literature. Expected symmetry for the final results is produced for the DMA method. The results obtained indicate that DMA holds significant potential in being able to accurately compute turbulent flow without modeling for practical engineering applications.

The present study has been performed to demonstrate proof of principle. Significantly more research should be pursued to fully explore the effectiveness and efficiency of the method. Research should be performed to investigate how best to initiate turbulence to minimize the initial DNS calculation, how long the DNS should be performed, how much to scale-up for a given scaling, which numerical methods are best (fastest and most accurate) to use to perform DMA, how well the method performs for large Reynolds number flows and how the method can be applied to complex geometries.

\section{References}

Bazilevs, Y., Calo, V. M., Cottrell, J. A., Hughes, T. J. R., Reali, A., and Scovazzi, G., 2007, Variational multiscale residual-based turbulence modeling for large eddy simulation of incompressible flows, Comput. Meth. App. Mech. \& Eng., 197, 173-201. 
Hughes, Thomas J. R., Feijóo, Gonzalo R., Mazzei, Luca, and Quincy, Jean-Baptiste, 1998, The variational multiscale method - a paradigm for computational mechanics, Comput. Meth. App. Mech. \& Eng., 166, 3-24.

Kemenov, K. A. and Menon, S., 2006, Explicit small-scale velocity simulation for high-Re turbulent flows, J. Comp. Physics, 220, 290-311.

Pinelli, A., Uhlmann, M., Sekimoto, A., and Kawahara, G., 2010, Reynolds number dependence of mean flow structure in square duct turbulence, J. Fluid Mech., 644, 107-122.

Raiesi, H., Pollard, A., and Piomelli, U., 2011, Direct Numerical Simulations of Turbulence Induced Secondary Motion in Square and Skewed Ducts, Seventh International Symposium on Turbulence and Shear Flow Phenomena (TSFP-7), July 28-31, Ottawa, Canada.

Sagaut, P., Deck, S. and Terracol, M., 2006, Multiscale and Multiresolution Approaches in Turbulence, Imperial College Press, London.

Wilcox, D. C., 1993, Turbulence Modeling for CFD, DCW Industries, Inc., La Cañada, California. 